\title{
PET/CT Image Artifacts Caused by the Arms
}

\author{
Ismet Sarikaya $^{1}$ and Ali Sarikaya ${ }^{2}$ \\ ${ }^{I}$ Department of Nuclear Medicine, Kuwait University Faculty of Medicine, Safat, Kuwait; and ${ }^{2}$ Department of Nuclear Medicine, \\ Trakya University Faculty of Medicine, Edirne, Turkey
}

\begin{abstract}
CE credit: For CE credit, you can access the test for this article, as well as additional JNMT CE tests, online at https://www.snmmilearningcenter.org. Complete the test online no later than March 2024. Your online test will be scored immediately. You may make 3 attempts to pass the test and must answer $80 \%$ of the questions correctly to receive $1.0 \mathrm{CEH}$ (Continuing Education Hour) credit. SNMMI members will have their CEH credit added to their VOICE transcript automatically; nonmembers will be able to print out a CE certificate upon successfully completing the test. The online test is free to SNMMI members; nonmembers must pay $\$ 15.00$ by credit card when logging onto the website to take the test.
\end{abstract}

PET/CT images are usually obtained in the arms-up position in patients with no head and neck pathology and in the arms-down position to image the head and neck area. The arms usually cause artifacts regardless of up or down positioning. These artifacts include beam hardening, scatter, truncation, and cold areas (cold artifacts) in obese or large patients; motion artifacts; implanted-metal-object artifacts; and artifacts related to radiotracer extravasation at the injection site. In this review article, we will discuss the mechanisms of these artifacts and suggest solutions to reduce or eliminate them, such as reviewing the non-attenuation-corrected PET images, performing extended-field-of-view reconstruction, not applying scatter correction, and using software to correct beam-hardening, scatter, and truncation artifacts. We will present various PET/CT images before and after corrections for such artifacts.

Key Words: PET/CT; artifacts; arms; truncation; cold artifacts

J Nucl Med Technol 2021; 49:19-22

DOI: $10.2967 /$ jnmt.120.248641

\section{$\mathbf{P}$} ET/CT images are usually obtained in the arms-up position if there is no head and neck pathology and in the armsdown position to image the brain or the head and neck area. When patients cannot raise their arms, artifacts in the body area may result, particularly if the patients are obese or large. However, whether up or down, the arms can cause artifacts (1). Because the area of interest is usually the body (chest, abdomen, or pelvis), artifacts in arms-down imaging are more of a concern than artifacts in arms-up imaging. These artifacts include beam hardening and scatter artifacts between the right and left arm bones, truncation artifacts, photopenic or cold areas (cold artifacts) in the soft tissues and bones in the abdomen and pelvis in obese or large patients, artifacts related to

Received Apr. 29, 2020; revision accepted Jun. 17, 2020.

For correspondence or reprints contact: Ismet Sarikaya, Department of Nuclear Medicine, Kuwait University, P.O. Box 24923, Safat, Kuwait 13110. E-mail: isarikaya99@yahoo.com

Published online Jul. 24, 2020

COPYRIGHT @ 2021 by the Society of Nuclear Medicine and Molecular Imaging. arm motion, artifacts caused by metal objects in the arms, and artifacts from extravasated activity at the injection site.

\section{BEAM HARDENING AND SCATTER}

Beam hardening is commonly seen on CT images. Beamhardening and scatter artifacts can occur between the right and left arm bones on CT images and may result in inadequate correlation of CT data with PET images, mimic pathologic conditions, or result in inaccurate Hounsfield units if the artifacts are severe (2-4). Although not causing significant image artifacts on PET images, beam hardening, if severe, may cause suboptimal attenuation correction (AC), a reduction in the detectability of small lesions, or inaccurate or suboptimal SUVs (Fig. 1). Beam-hardening and scatter artifacts are produced by different mechanisms but appear as multiple linear bands or streaks between two highattenuation objects (bone, metal, or barium) on CT images (Fig. 1) (3). When the x-ray beam passes through the body, low-energy $x$-ray photons are attenuated more easily than high-energy photons, which is a particular problem with high atomic number objects (bone, iodine, or metal) (5). The effects of beam hardening on the image can be reduced using iterative reconstruction or with beam-hardening and scatter-correction software $(5,6)$.

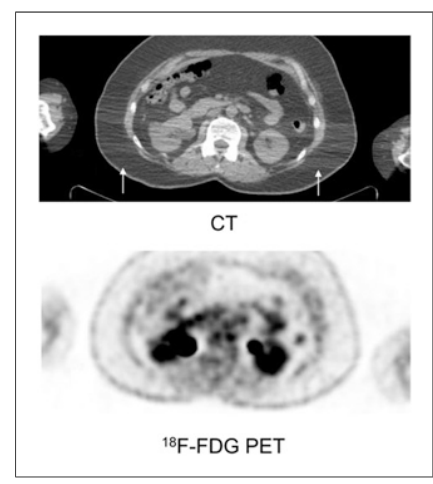

FIGURE 1. Selected transaxial CT image shows beam-hardening artifact between arms (arrows), with no artifact seen on PET image. 


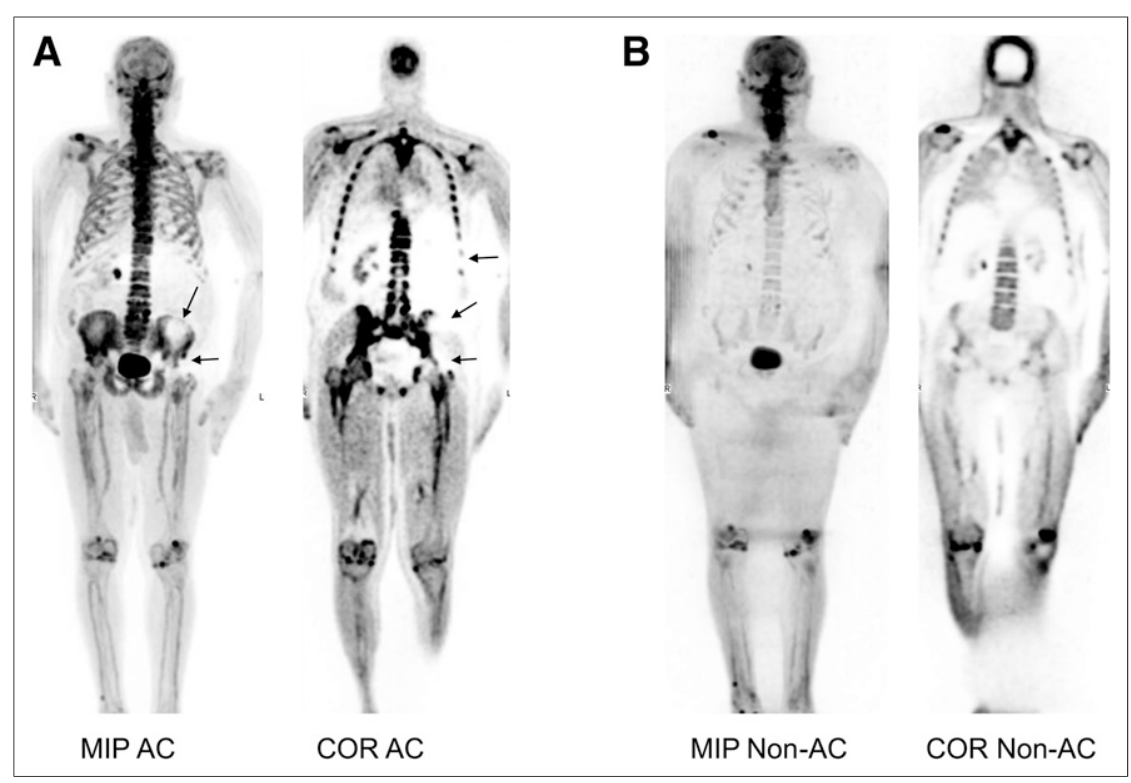

FIGURE 2. Whole-body coronal (COR) and maximum-intensity projection (MIP) AC ${ }^{18} \mathrm{~F}-\mathrm{NaF}$ PET/CT images of large patient who was imaged with arms down. (A) AC PET images demonstrate large cold area (cold artifact) on left side of abdomen and pelvis, with reduced uptake in bones (left lower ribs, hemipelvis, and hip) and soft tissues (left kidney). Left arm is farther from body than right arm. (B) This cold artifact is not seen on non-AC PET images.

\section{TRUNCATION AND COLD ARTIFACTS}

Obtaining images in the arms-down position in obese or large patients usually causes truncation artifacts (7). The fact that the CT field of view (FOV) is smaller than the PET FOV in large patients (object extending beyond

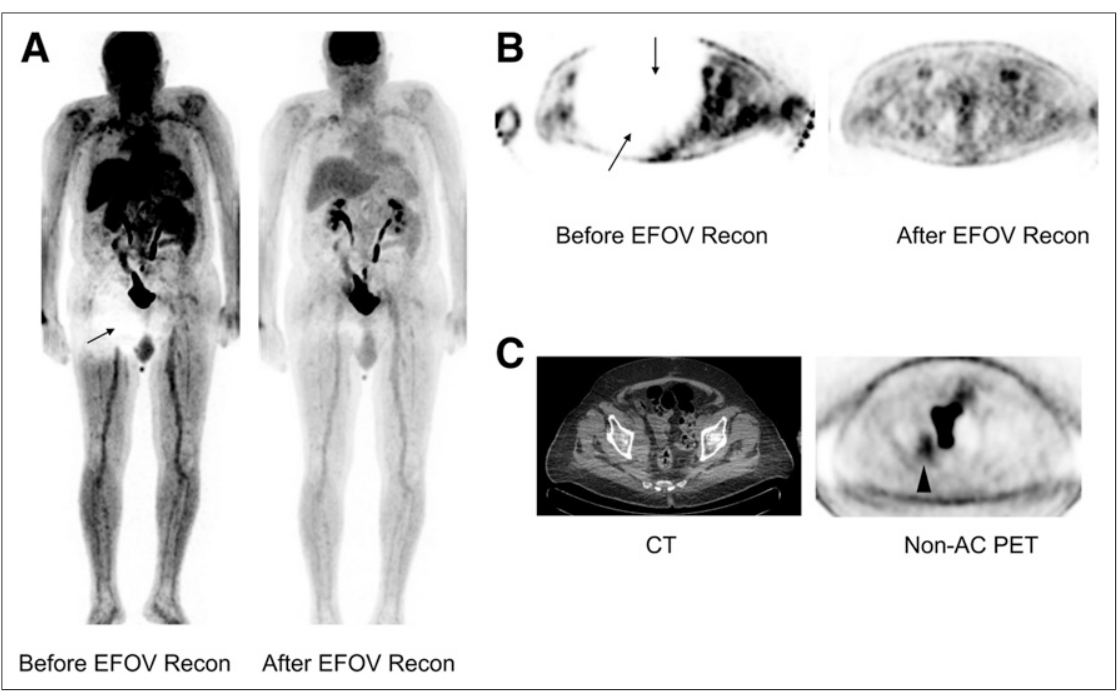

FIGURE 3. (A and B) Whole-body maximum-intensity projection $(A)$ and transaxial pelvic (B) ${ }^{18} \mathrm{~F}-F D G$ PET AC images before and after extended-FOV reconstruction (EFOV recon). Cold artifact in right hip and pelvis (arrows) disappears after extended FOV reconstruction. Right arm is farther from body than left arm. (C) Cold artifact is also not present on non-AC PET image. Right pelvic hypermetabolic mass (arrowhead) on transaxial CT and non-AC PET images was missed on AC PET image because of cold artifact. (Note that images in B and $C$ are not from the same level.) the scanning field of view on CT) may cause inaccurate $\mathrm{AC}(8,9)$. In the arms-down position, the body of the patient extends beyond the transverse CT FOV and creates truncation that is extensive in obese or large patients $(1)$.

In truncation artifacts, at the periphery of the images there is non-AC PET data without corresponding $\mathrm{CT}$ images $(2,7)$. To minimize truncation artifacts in the body, patients can be imaged with their arms up. A truncation-correction algorithm or an extended-FOV reconstruction (extending the reconstruction field of view) can correct these artifacts (2,9-11). Newer scanners with larger CT FOVs can reduce truncation artifacts.

The cold artifacts seen on AC PET images (in the abdominal and pelvic regions in obese or large patients imaged in the arms-down position or in the head and neck region in patients imaged in the hyperflexed arms-up position) are due to extensive truncation or are the result of scatter correction. These cold artifacts are not seen on non-AC PET images, after extended FOV reconstruction, after removing the arms from the imaging FOV, or when not applying scatter correction (Figs. 2-6) $(1,12)$. Cold artifacts seen on only one side of the body are likely due to noncentral placement of the patient on the imaging table or to flexing of one arm more than the other, causing unilateral truncation (Figs. 2-4). Keeping the arms straight during the image acquisition and placing the patient symmetrically on the table can reduce these artifacts. Non-AC PET images can grossly assess the areas but usually provide inadequate information in obese or large patients, particularly in deep tissues. When extended-FOV reconstruction is available, it successfully eliminates these cold areas. Scatter correction may induce cold artifacts when it is applied with an iterative reconstruction (scatter-correction artifact), particularly when the patients are scanned with their arms down and the hands and fingers away from the body, resulting in photopenic or cold areas at the level of the hands (12). Changing the type of scatter correction (from relative to absolute) or not using scatter correction can eliminate these artifacts (1214). Scatter-correction artifacts can also 


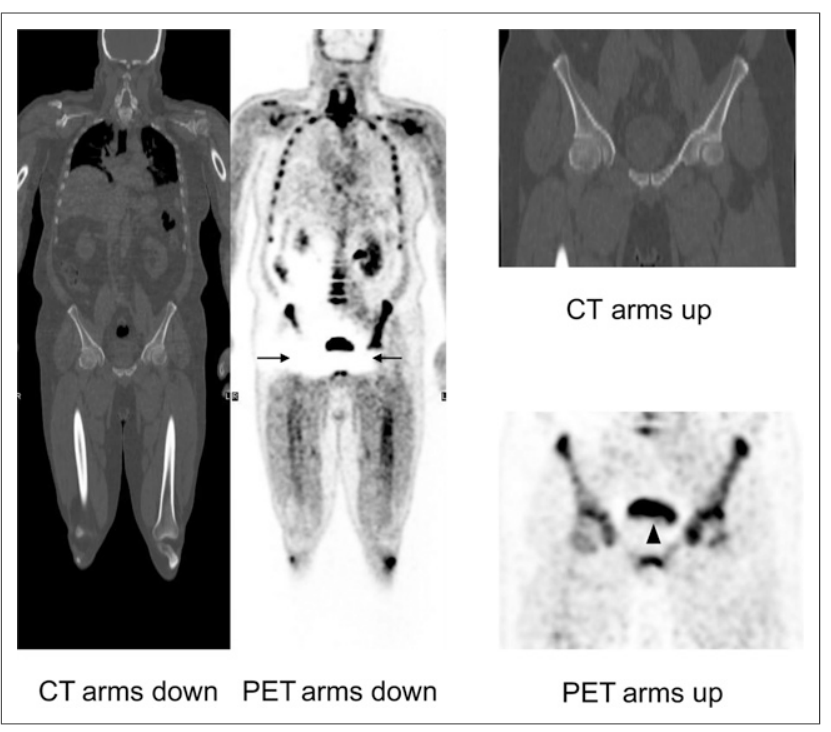

FIGURE 4. Whole-body coronal CT (left and top) and AC ${ }^{18} \mathrm{~F}-$ $\mathrm{NaF}$ PET (right and bottom) images of large patient. Cold artifact (arrows) is seen in hips (more significant on right side) on PET image obtained with arms down. Right arm is farther from body than left arm. This cold defect is not seen after repeating PET/CT with arms up. Note the enlarged prostate elevating bladder (arrowhead).

be seen around the kidneys and bladder as photopenic areas (a halo). There are some inaccurate scatter-correction methods in current use in clinical PET imaging that tend to overestimate the scatter contribution (14).

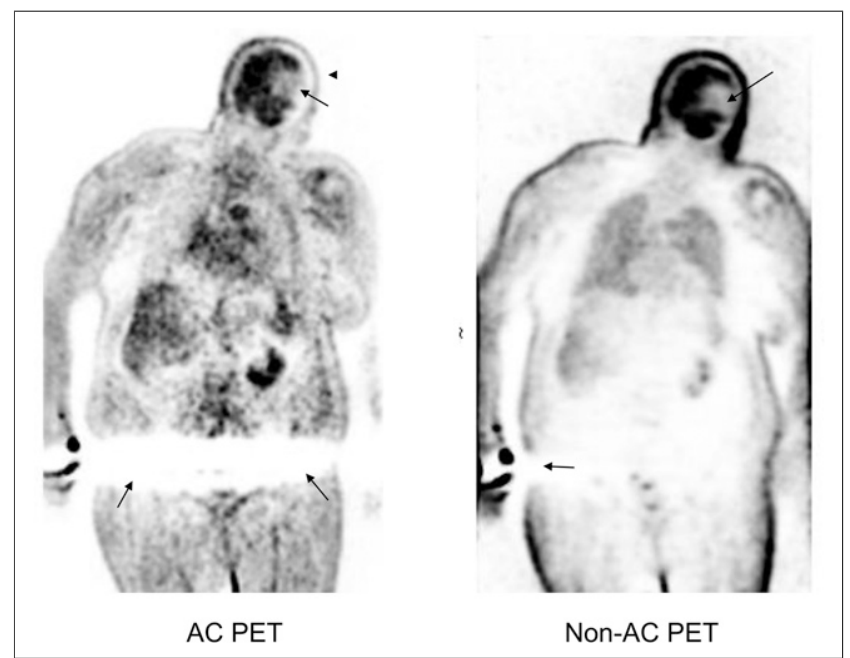

FIGURE 5. Whole-body coronal AC ${ }^{18} \mathrm{~F}-\mathrm{FDG}$ PET image demonstrates linear cold artifact (bottom arrows) in pelvis of large patient imaged with arms down. This artifact is not present on non-AC PET image. Small cold artifact (bottom arrow) in skin adjacent to right arm on non-AC PET image is scatter-correction artifact due to activity extravasation at injection site. Defect on left side of brain (arrows) on both AC and non-AC images was caused by cerebral infarct (seen on CT image; not shown). Reduced uptake in scalp (arrowhead), seen on AC image only, was caused by head motion after acquiring CT scan.

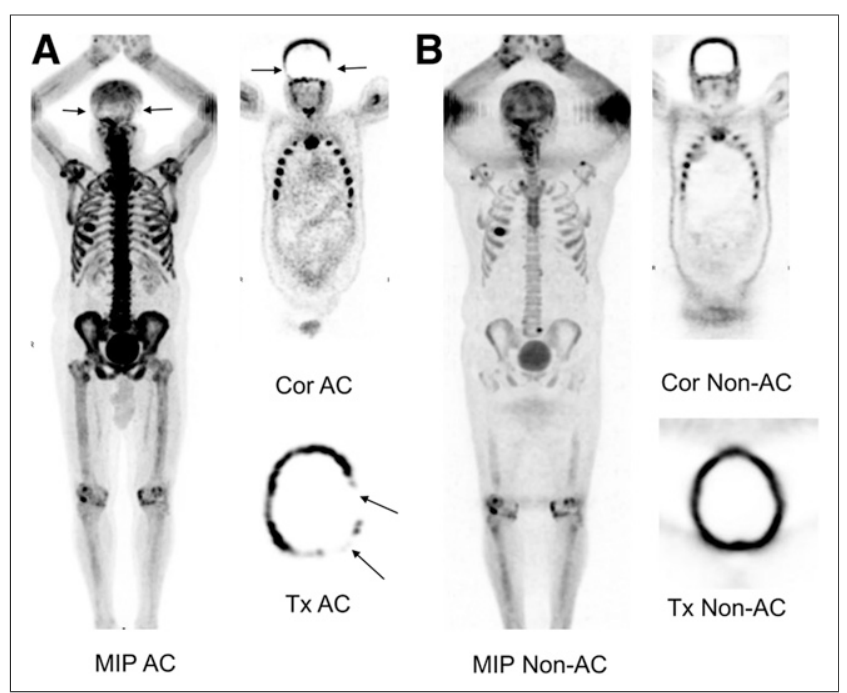

FIGURE 6. (A) Whole-body maximum-intensity projection (MIP) and coronal (Cor) and transaxial (Tx) AC ${ }^{18} \mathrm{~F}-\mathrm{NaF}$ PET images demonstrate linear cold artifact in skull, more prominent on left (arrows). (B) Artifact is not present on non-AC images.

\section{OTHER ARTIFACTS RELATED TO THE ARMS}

The other artifacts caused by the arms are related to arm motion, metal objects in the arms, and extravasated activity at the injection site.

Arm motion can cause cold artifacts (areas of washedout activity) and distorted arm anatomy on PET images (Fig. 7A) $(15,16)$. Such artifacts can affect visual and quantitative assessment of PET uptake. Reviewing the non-AC PET images can help if the motion occurred after the $\mathrm{CT}$ acquisition and was not continuous during the PET acquisition. Scatter limitation (no scatter correction) can

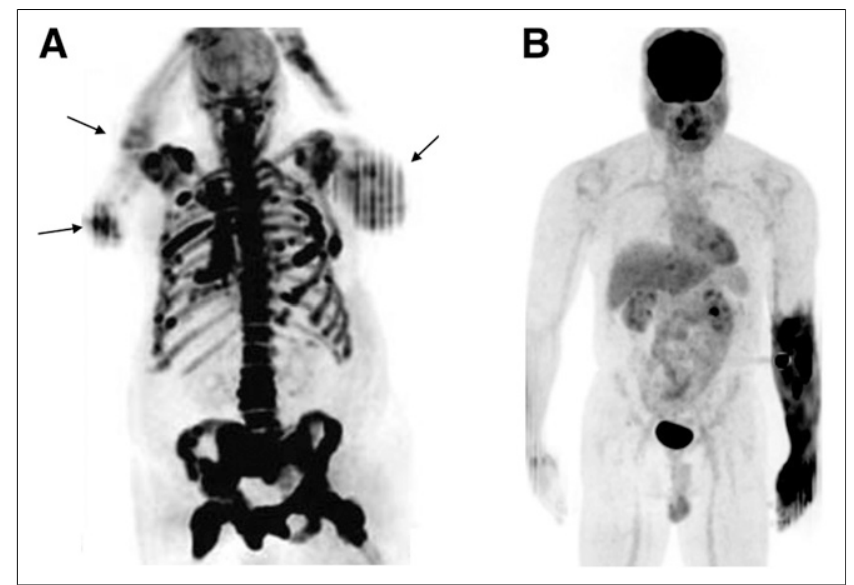

FIGURE 7. (A) ${ }^{18} \mathrm{~F}-\mathrm{NaF}$ PET maximum-intensity projection (MIP) image demonstrates arm motion artifacts bilaterally (arrows). (B) Accidental intraarterial injection of radiotracer caused diffusely increased uptake in tissues of left forearm and hand seen on ${ }^{18}$ F-FDG PET maximum-intensity projection image. 
correct overestimation of scatter caused by arm motion $(15,16)$.

Metal objects in the arms can cause beam-hardening and scatter artifacts. Metal-artifact reduction and metal-deletion techniques can reduce these artifacts (3).

Radiotracer extravasation at the injection site causes various artifacts, such as reconstruction and scatter-correction artifacts that may obscure the adjacent tissues, a hot spot that may mimic a lesion at the injection site, lymph node uptake that may be mistaken for metastasis, and an erroneously low SUV and low uptake in tissues and lesions if extravasation is high (Fig. 5). Accidental intraarterial injection of radiotracer can cause diffusely increased uptake in the tissues distal to the injection site (glove pattern) and may result in underestimation of lesion SUV and inadequate assessment of the arm (Fig. 7B) (17). Performing proper injection technique, removing the arm from the imaging FOV, or using iterative reconstruction can help to reduce or eliminate these artifacts.

\section{CONCLUSION}

For accurate interpretation of PET/CT images, it is impor tant to understand and detect certain artifacts. In this review article, we have summarized the PET/CT artifacts caused by the arms-down and arms-up positions and suggested solutions to reduce or avoid these artifacts.

\section{DISCLOSURE}

No potential conflict of interest relevant to this article was reported.

\section{REFERENCES}

1. Sarikaya I, Elgazzar AH, Sarikaya A, et al. Normal bone and soft tissue distribution of fluorine-18-sodium fluoride and artifacts on ${ }^{18} \mathrm{~F}-\mathrm{NaF}$ PET/CT bone scan: a pictorial review. Nucl Med Commun. 2017;38:810-819.

2. Simpson DL, Bui-Mansfield LT, Bank KP. FDG PET/CT: artifacts and pitfalls. Contemp Diagn Radiol. 2017;50:1-8.

3. Boas EF, Fleischmann D. CT artifacts: causes and reduction techniques. Imaging Med. 2012;4:229-240.

4. Blodgett TM, Mehta AS, Mehta AS, et al. PET/CT artifacts. Clin Imaging. 2011;35:49-63.

5. Barrett JF, Keat N. Artifacts in CT: recognition and avoidance. Radiographics. 2004;24:1679-1691.

6. Popilock R, Sandrasagaren K, Harris L, et al. CT artifact recognition for the nuclear technologist. J Nucl Med Technol. 2008;36:79-81.

7. Mawlawi O, Erasmus JJ, Pan T, et al. Truncation artifact on PET/CT: impact on measurements of activity concentration and assessment of a correction algorithm. AJR. 2006;186:1458-1467.

8. Townsend DW, Beyer Th, Blodgett TM. PET/CT scanners: a hardware approach to image fusion. Semin Nucl Med. 2003;33:193-204.

9. Zamyatin AA, Nakanishi S. Extension of the reconstruction field of view and truncation correction using sinogram decomposition. Med Phys. 2007;34:1593-1604.

10. Schaller S, Sembritzki O, Beyer T, Fuchs T, Kachelriess M, Flohr T. An algorithm for virtual extension of the CT field of measurement for application in combined PET/CT scanners. Radiology. 2002;225:497-499.

11. Kunze H, Härer W, Stierstorfer K. Iterative extended field of view reconstruction. In: Medical Imaging 2007: Physics of Medical Imaging. SPIE; 2007:65105X.

12. PET/CT Atlas on Quality Control and Image Artefacts. International Atomic Energy Agency; 2014:30:41-44.

13. Watson CC. New, faster, image-based scatter correction for 3-D PET. IEEE Trans Nucl Sci. 2000;47:1587-1594.

14. Heußer T, Mann P, Rank CM, et al. Investigation of the halo-artifact in ${ }^{68} \mathrm{Ga}-$ PSMA-11-PET/MRI. PLoS One. 2017; 12:e0183329.

15. Lodge MA, Mhlanga JC, Cho SY, et al. Effect of patient arm motion in wholebody PET/CT. J Nucl Med. 2011;52:1891-1897.

16. Callahan J, Binns D, Deller T, et al. Scatter limitation to correct for arm movement in PET/CT. Clin Nucl Med. 2012;37:786-787.

17. Zhu Z, Doss M, Tan H, et al. Inadvertent intraarterial injection of ${ }^{18}$ F-FDG: a case report and literature review of hot forearm and hot hand signs. J Nucl Med Technol. 2011;39:249-251. 